Canad. Math. Bull. Vol. 50 (4), 2007 pp. 637-640

\title{
Index of Volume 50
}

A 161 Arapura, Donu, Su-Jeong Kang Functoriality of the Coniveau Filtration

172 Aron, Richard, Pamela Gorkin An Infinite Dimensional Vector Space of Universal Functions for $H^{\infty}$ of the Ball

B 3 Basener, Richard F. Higher Dimensional Spaces of Functions on the Spectrum of a Uniform Algebra

321 Blair, David E. On Lagrangian Catenoids

481 Blanloil, Vincent, Osamu Saeki Concordance des nœuds de dimension 4

11 Borwein, David, Jonathan Borwein van der Pol Expansions of L-Series

11 Borwein, Jonathan See David Borwein

24 Brown, Nathan, Rachel Finck, Matthew Spencer, Kristopher Tapp, Zhongtao Wu Invariant Metrics with Nonnegative Curvature on Compact Lie Groups

C 182 Chapoton, Frédéric On the Coxeter Transformations for Tamari Posets

334 Chiang-Hsieh, Hung-Jen, Yifan Yang Determination of Hauptmoduls and Construction of Abelian Extensions of Quadratic Number Fields

D 347 de Dios Pérez, Juan, Florentino G. Santos, Young Jin Suh Real Hypersurfaces in Complex Projective Space Whose Structure Jacobi Operator Is of Codazzi Type

191 Drungilas, Paulius, Artūras Dubickas Every Real Algebraic Integer Is a Difference of Two Mahler Measures

191 Dubickas, Artūras See Paulius Drungilas

504 Dukes, Peter, Alan C. H. Ling Asymptotic Existence of Resolvable Graph Designs

434 Duman, Oktay See M. Ali Özarslan

35 Duyckaerts, Thomas A Singular Critical Potential for the Schrödinger Operator

48 Dvorsky, Alexander Tensor Square of the Minimal Representation of $O(p, q)$

F 196 Fernández, Julio, Josep González, Joan-C. Lario Plane Quartic Twists of X(5, 3)

356 Filippakis, Michael E., Nikolaos S. Papageorgiou Existence of Positive Solutions for Nonlinear Noncoercive Hemivariational Inequalities

24 Finck, Rachel See Nathan Brown

G 365 Godinho, Leonor Equivariant Cohomology of $S^{1}$-Actions on 4-Manifolds

206 Golasinski, Marek, Daciberg Lima Gonçalves Spherical Space Forms: Homotopy Types and Self-Equivalences for the Group $(\mathbb{Z} / a \rtimes \mathbb{Z} / b) \times S L_{2}\left(\mathbb{F}_{p}\right)$ 
206 Gonçalves, Daciberg Lima See Marek Golasiński

196 González, Josep See Julio Fernández

172 Gorkin, Pamela See Richard Aron

56 Gourdeau, F., A. Pourabbas, M. C. White Simplicial Cohomology of Some Semigroup Algebras

71 Gurak, S. Polynomials for Kloosterman Sums

377 Gutierrez, C., X. Jarque, J. Llibre, M. A. Teixeira Global Injectivity of $C^{1}$ Maps of the Real Plane, Inseparable Leaves and the Palais-Smale Condition

H 85 Han, Deguang Classification of Finite Group-Frames and Super-Frames

390 Hebda, James J., Chun-Chung Hsieh, Chichen M. Tsau Linking Number of Singular Links and the Seifert Matrix

519 Henson, C. Ward, Yves Raynaud, Andrew Rizzo On Axiomatizability of Non-Commutative $L_{p}$-Spaces

535 Hohlweg, Christophe Generalized Descent Algebras

390 Hsieh, Chun-Chung See James J. Hebda

I 547 Iakovlev, Serguei Inverse Laplace Transforms Encountered in Hyperbolic Problems of Non-Stationary Fluid-Structure Interaction

J 377 Jarque, X. See C. Gutierrez

567 Joshi, Kirti Exotic Torsion, Frobenius Splitting and the Slope Spectral Sequence

K 486 K. Hulek See S. Cynk

161 Kang, Su-Jeong See Donu Arapura

97 Kim, In-Bae, Ki Hyun Kim, Woon Ha Sohn Characterizations of Real Hypersurfaces in a Complex Space Form

97 Kim, Ki Hyun See In-Bae Kim

105 Klep, Igor On Valuations, Places and Graded Rings Associated to $*$-Orderings

215 Kloosterman, Remke Elliptic K3 Surfaces with Geometric Mordell-Weil Rank 15

399 Komornik, Vilmos, Paola Loreti Expansions in Complex Bases

579 Kot, Piotr $p$-Radial Exceptional Sets and Conformal Mappings

227 Kucerovsky, D., P. W. Ng AF-Skeletons and Real Rank Zero Algebras with the Corona Factorization Property

234 Kuo, Wentang A Remark on a Modular Analogue of the Sato-Tate Conjecture

L 588 Labute, John, Nicole Lemire, Ján Mináč, John Swallow Cohomological Dimension and Schreier's Formula in Galois Cohomology

243 Langlands, Robert $P$. Un nouveau point de repère dans la théorie des formes automorphes 
196 Lario, Joan-C. See Julio Fernández

594 Laubie, François Ramification des groupes abéliens d'automorphismes des corps $\mathbb{F}_{q}((X))$

588 Lemire, Nicole See John Labute

113 Li, ZhenYang, Xi Zhang Hermitian Harmonic Maps into Convex Balls

504 Ling, Alan C. H. See Peter Dukes

377 Llibre, J. See C. Gutierrez

598 Lorestani, Keivan Borna, Parviz Sahandi, Siamak Yassemi Artinian Local Cohomology Modules

399 Loreti, Paola See Vilmos Komornik

409 Luca, Florian, Igor E. Shparlinski Discriminants of Complex Multiplication Fields of Elliptic Curves over Finite Fields

M 268 Manuilov, V., K. Thomsen On the Lack of Inverses to $C^{*}$-Extensions Related to Property T Groups

418 Matui, Hiroki A Short Proof of Affability for Certain Cantor Minimal $\mathbb{Z}^{2}$-Systems

284 McIntosh, Richard J. Second Order Mock Theta Functions

588 Mináč, Ján See John Labute

427 Moreno Mejía, Israel On the Image of Certain Extension Maps. I

N 227 Ng, P. W. See D. Kucerovsky

123 Nikolov, Nikolai, Peter Pflug Simultaneous Approximation and Interpolation on Arakelian Sets

O 126 Ongay, Fausto $\varphi$-Dialgebras and a Class of Matrix "Coquecigrues"

434 Özarslan, M. Ali, Oktay Duman MKZ Type Operators Providing a Better Estimation on $[1 / 2,1)$

P 356 Papageorgiou, Nikolaos S. See Michael E. Filippakis

603 Penkov, Ivan, Gregg Zuckerman Construction of Generalized Harish-Chandra Modules with Arbitrary Minimal 1 -Type

123 Pflug, Peter See Nikolai Nikolov

56 Pourabbas, A. See F. Gourdeau

R 440 Raghuram, A. A Künneth Theorem for $p$-Adic Groups

519 Raynaud, Yves See C. Ward Henson

519 Rizzo, Andrew See C. Ward Henson

610 Rychtář, Jan, Jiří Spurný On Weak* Kadec-Klee Norms 
S 486 S. Cynk, K. Hulek Higher-Dimensional Modular Calabi-Yau Manifolds

481 Saeki, Osamu See Vincent Blanlœil

598 Sahandi, Parviz See Keivan Borna Lorestani

347 Santos, Florentino G. See Juan de Dios Pérez

138 Sari, Bünyamin On the Structure of the Set of Symmetric Sequences in Orlicz Sequence Spaces

291 Sarkar, Rudra P., Jyoti Sengupta Beurling's Theorem and Characterization of Heat Kernel for Riemannian Symmetric Spaces of Noncompact Type

291 Sengupta, Jyoti See Rudra P. Sarkar

409 Shparlinski, Igor E. See Florian Luca

149 Śliwa, Wiesław On Quotients of Non-Archimedean Köthe Spaces

447 Śniatycki, Jędrzej Generalizations of Frobenius' Theorem on Manifolds and Subcartesian Spaces

97 Sohn, Woon Ha See In-Bae Kim

24 Spencer, Matthew See Nathan Brown

460 Spielberg, Jack Weak Semiprojectivity for Purely Infinite $C^{*}$-Algebras

610 Spurný, Jiři See Jan Rychtár

347 Suh, Young Jin See Juan de Dios Pérez

588 Swallow, John See John Labute

T 24 Tapp, Kristopher See Nathan Brown

619 Tcaciuc, Adi On the Existence of Asymptotic- $l_{p}$ Structures in Banach Spaces

377 Teixeira, M. A. See C. Gutierrez

268 Thomsen, K. See V. Manuilov

158 Tipu, Vicentiu A Note on Giuga's Conjecture

390 Tsau, Chichen M. See James J. Hebda

469 Tvalavadze, M. V. Simple Decompositions of the Exceptional Jordan Algebra

313 Tzermias, Pavlos On Cauchy-Liouville-Mirimanoff Polynomials

W 56 White, M. C. See F. Gourdeau

$24 \mathrm{Wu}$, Zhongtao See Nathan Brown

Y 334 Yang, Yifan See Hung-Jen Chiang-Hsieh

598 Yassemi, Siamak See Keivan Borna Lorestani

Z 632 Zelenyuk, Yevhen, Yuliya Zelenyuk Transformations and Colorings of Groups

632 Zelenyuk, Yuliya See Yevhen Zelenyuk

113 Zhang, Xi See ZhenYang Li

474 Zhou, Jiazu On Willmore's Inequality for Submanifolds

603 Zuckerman, Gregg See Ivan Penkov 